\title{
Efeitos do tratamento com realidade virtual não imersiva na qualidade de vida de indivíduos com Parkinson
}

\author{
Effects of treatment with non-immersive virtual reality in the quality of life of people with \\ Parkinson's disease
}

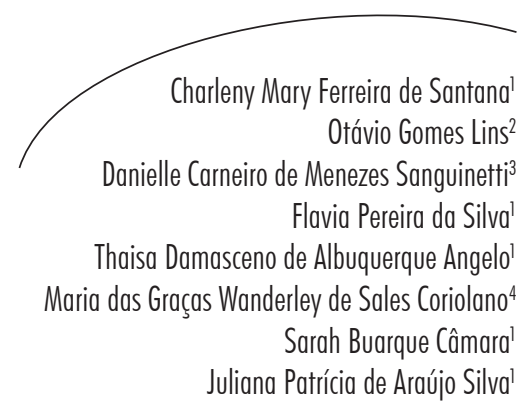

\section{Resumo}

O objetivo desta pesquisa foi verificar os efeitos do tratamento com realidade virtual não imersiva na qualidade de vida de indivíduos com doença de Parkinson, antes e após o tratamento com aplicação do questionário da doença de Parkinson (PDQ-39). Corresponde a um estudo experimental, do tipo ensaio clínico não controlado, de natureza quantitativa. O estudo envolveu 14 indivíduos, que passaram por 20 sessões individuais de tratamento distribuídas em duas sessões semanais, com duração entre 30 e 40 minutos cada. Na análise dos dados, foi utilizado o teste de Wilcoxon, para encontrar o valor da significância estatística $(\mathrm{p} \leq 0,05)$. Os escores do Questionário da Doença de Parkinson-39 total e seus domínios diminuíram quando comparados aos do período da avaliação e da reavaliação dos pacientes. No entanto, os valores de p expressam que essa diminuição foi significativa especificamente para os domínios mobilidade, bem-estar emocional, estigma e cognição, e o escore do Questionário da Doença de Parkinson-39 total. Assim, os resultados demonstraram que a realidade virtual não imersiva contribuiu positivamente sobre a qualidade de vida desses indivíduos.

\footnotetext{
Universidade Federal de Pernambuco. Centro de Ciências da Saúde, Departamento de Terapia Ocupacional. Recife, PE, Brasil.

2 Universidade Federal de Pernambuco. Centro de Ciências da Saúde, Departamento de Neuropsiquiatria. Recife, PE, Brasil.

3 Universidade Federal da Paraíba. Centro de Ciências da Saúde, Departamento de Terapia Ocupacional. João Pessoa, PB, Brasil.

4 Universidade Federal de Pernambuco. Centro de Ciências Biológicas, Departamento de Anatomia Humana. Recife, PE, Brasil.
}

Financiamento: Fundação de Amparo à Ciência e Tecnologia do Estado de Pernambuco -FACEPE, processo $n^{\circ}$ BIC-0052-4.01/11, por meio de concessão de bolsa para o Programa de Iniciação Científica.

Esse trabalho é um recorte da pesquisa do doutorado de Danielle Sanguinetti intitulada "Uso de Jogos em Realidade Virtual Não Imersiva no Tratamento de Pessoas com Doença de Parkinson”.

Palavras-chave: Realidade Virtual. Doença de Parkinson. Qualidade de Vida. Terapia Ocupacional. 


\section{Abstract}

This research aimed to investigate the effects of treatment with non-immersive virtual reality in the quality of life of individuals with Parkinson's disease before and after treatment with the questionnaire of Parkinson's disease (PDQ-39). It corresponds to an experimental study, quantitative, of uncontrolled clinical trial type. It involved 14 individuals who underwent 20 individual treatment sessions divided into two weekly sessions lasting 30 to 40 minutes each. In the data analysis, the Wilcoxon test was used to find the value of statistical significance $(p=0.05)$. The scores of Questionnaire Parkinson's disease-39 overall and its domains decreased when compared to the evaluation period and the revaluation of patients. However, $\mathrm{p}$ values express that this decrease was significant if specific areas for mobility, emotional well-being, stigma and cognition, and the Questionnaire score of Parkinson's Disease-39 overall. Thus, the results demonstrated that non-immersive virtual reality contributed positively on the quality of life of these individuals.
Key words: Virtual Reality. Parkinson Disease. Quality of Life. Occupational Therapy.

\section{INTRODUÇÃO}

A doença de Parkinson (DP) é uma doença neurológica de caráter crônico-degenerativa progressiva que acomete um em cada mil indivíduos da população geral. ${ }^{1,2}$ A maioria dos casos apresenta-se na faixa etária dos 50 aos 70 anos de idade, sendo mais homens que mulheres. ${ }^{3}$ A estimativa mundial é que em 2020 mais de 40 milhões de pessoas apresentem desordens motoras secundárias à $\mathrm{DP}$, devido ao aumento da população de pessoas idosas. ${ }^{4}$

Sua etiologia é tida como idiopática, porém alguns autores relacionam seu surgimento a um conjunto de possíveis causas, tais como: fatores genéticos, toxinas ambientais, estresse oxidativo, anormalidades mitocondriais e/ou alterações do envelhecimento. Essas causas podem acarretar diminuição da dopamina na via negroestriatal, por morte dos neurônios motores da substância negra, responsáveis pela precisão, uniformidade dos movimentos e coordenação das mudanças posturais. ${ }^{2,3,5,6}$

Outros autores relacionam o surgimento com o comprometimento de outros sistemas monoaminérgicos, como os neurotransmissores serotonérgicos e o noradrenérgicos. Por conseguinte, o comprometimento desses outros sistemas poderia explicar o surgimento dos sintomas não motores, que tornam a DP ainda mais incapacitante. ${ }^{2,7,8}$ Os sintomas motores cardinais são: tremor em repouso, rigidez muscular, bradicinesia (lentificação da ação do movimento) e disfunções posturais. ${ }^{2,6-8}$

Dentre os sintomas não motores estão disfunções neuropsiquiátricas, distúrbios do sono, depressão, algumas disfunções sensoriais e alterações cognitivas, como dificuldades de concentração, atenção, alterações nas funções executivas e memória recente, dificuldades no raciocínio (cálculos) e em atividades que requerem orientação espacial. ${ }^{2,7,8}$

A progressão da doença está marcada pelo comprometimento da condição física, resultando na diminuição da independência funcional. Com o agravamento dos sintomas motores e não motores, poderá ocorrer prejuízo na qualidade de vida $(\mathrm{QV}) .^{3}$

QV pode ser considerado um termo multidimensional, que avalia a satisfação subjetiva em relação à expectativa de vida e outros aspectos, como relações sociais, saúde, questões financeiras, trabalho, condições de moradia, transporte, níveis de poluição, independência, educação, religião, atividades de lazer. ${ }^{1,9-12}$ 
No contexto da prática clínica, surge ainda o termo "qualidade de vida relacionada à saúde" (QVRS), que corresponde à relação da QV com os aspectos afetados direta ou indiretamente por uma doença e/ou tratamento, considerando a percepção do indivíduo sobre os aspectos comprometidos e as repercussões na própria vida, incluindo a satisfação pessoal associada a seu bem-estar físico, funcional, emocional e social. ${ }^{1,12}$

Dessa forma, o principal foco do tratamento em indivíduos com DP deve ser a manutenção ou recuperação da $Q V,,^{13,14}$ o que enfatiza a importância de serem conhecidas as dimensões que abrangem os conceitos de QV e QVRS e os instrumentos de medida que avaliam o impacto sobre essas dimensões, pois estas informações podem auxiliar na escolha entre diferentes tratamentos e no monitoramento dos resultados das intervenções. ${ }^{1,9,10,12,13}$

A cada dia é mais consolidado o uso das tecnologias da área da informática aplicada à saúde, ampliando as possibilidades de estudo e aplicação de técnicas e procedimentos terapêuticos. ${ }^{15,16}$ A realidade virtual (RV) é uma nova maneira de interação homem-máquina, que propicia a movimentação, a visualização dos movimentos, interação em tempo real com personagens, atividades e ambientes tridimensionais, criados pelo computador, mas com representações cada vez mais próximas da realidade. ${ }^{15-17}$

Os sentidos da visão e audição costumam ser preponderantes em aplicações de RV, mas os outros sentidos - tato, vestibular, entre outros, como também habilidades e conhecimentos intuitivos - podem ser usados para enriquecer a experiência do usuário durante a interação com o mundo virtual. ${ }^{17,18}$

A realidade virtual não imersiva (RVNI) corresponde ao tipo de interação na qual o usuário é parcialmente transportado para o mundo virtual por uma janela, exemplo, um monitor, mas continua a sentir-se predominantemente no mundo real. ${ }^{19}$
Segundo Pompeu et al., ${ }^{20}$ o tratamento com RV poderá promover maior interação das habilidades motoras e cognitivas simultaneamente, o que é exigido pela maioria das atividades de vida diária (AVD). Assim, contribuirá para maior independência nas AVDs em comparação com o treino baseado apenas em estímulos motores. Dessa forma, o tratamento com a RV possivelmente causará efeitos positivos na $Q V$, visto que esta sofre influências negativas da progressão dos sintomas e da diminuição da independência funcional. ${ }^{3,20}$

O objetivo da pesquisa foi verificar os efeitos do tratamento com realidade virtual não imersiva na qualidade de vida de indivíduos com doença de Parkinson, com aplicação do questionário da doença de Parkinson (PDQ-39), antes e após o tratamento.

\section{MATERIAIS E MÉTODOS}

\section{Participantes}

Trata-se de um estudo experimental do tipo ensaio clínico não controlado, de natureza quantitativa. A pesquisa foi realizada entre agosto de 2011 e agosto de 2012. Os pacientes voluntários eram atendidos no ambulatório de neurologia do Hospital das Clínicas da Universidade Federal de Pernambuco (HCUFPE), pertencentes ao Programa PróParkinson (Programa de Extensão que promove assistência multiprofissional ao paciente com doença de Parkinson do HC/UFPE).

A equipe condutora da pesquisa foi composta por terapeutas ocupacionais. Foram incluídos pacientes com diagnóstico clínico de DP idiopática, atestada pelo neurologista chefe do serviço, nos estágios de Hoehn \& Yahr (I ao IV), de ambos os gêneros, com disponibilidade de irem até o local da pesquisa duas vezes por semana e que tinham capacidade para responder aos questionamentos dos instrumentos de avaliação de maneira coerente e compreender os comandos dados sobre o manejo, interação com os equipamentos e mais os jogos da pesquisa. 
Foram excluídos os pacientes com outra doença neurológica ou psiquiátrica associadas à DP, nível de Hoehn \& Yahr V.

\section{Instrumentos de avaliação}

\section{Escala de Estadiamento de Hoebn \& Yabr (HY)}

É um método rápido, bastante utilizado e prático, para determinar o estágio da doença. Neste estudo, foi utilizada em sua forma original, que consiste em cinco estágios de classificação, que avalia a severidade da DP e classifica o indivíduo quanto ao nível de incapacidade. Os pacientes classificados nos estágios I, II, e III apresentam incapacidade leve a moderada; os estágios IV e $\mathrm{V}$ apresentam pacientes com incapacidade mais grave. ${ }^{11}$

A aplicação da escala serviu para auxiliar na escolha e graduação dos jogos, considerando capacidades, habilidades e dificuldades dos pacientes, através da análise da atividade, técnica exclusiva de intervenção do terapeuta ocupacional. Essa técnica corresponde a uma análise minuciosa da ação humana, a qual determina o ponto de partida para o tratamento. Além disso, consiste não somente da análise de partes de uma atividade, mas de quais efeitos são gerados no paciente ao realizar a mesma. ${ }^{21}$

\section{Questionário da Doença de Parkinson (PDQ-39)}

É um instrumento considerado válido e sensível às mudanças vivenciadas pelas pessoas com DP. Tem sido indicado como o instrumento mais apropriado para a avaliação da QV desses pacientes. Foi desenvolvido pelo Departamento de Saúde Pública da Universidade de Oxford na Inglaterra. Já foi traduzido para diversas línguas, inclusive para português do Brasil, validado em vários países e sua possível utilização em estudos transculturais foi comprovada. ${ }^{1,9,22,23}$

É autoadministrável, composto por 39 questões subdivididas em oito domínios: mobilidade (dez questões), atividade de vida diária (seis questões), bem-estar emocional (seis questões), estigma (quatro questões), suporte social (três questões), cognição (quatro questões), comunicação (três questões) e desconforto corporal (três questões). ${ }^{1,9,13,23}$

Para cada questão, há cinco opções diferentes de resposta (nunca; raramente; algumas vezes; frequentemente e sempre), com a pontuação variando de 0 a 4 , conforme a ordem das respostas supracitadas. As respostas referem-se ao impacto da doença sobre a vida do paciente em relação ao último mês vivido, conforme deve ser explicado antes da avaliação. Os escores do instrumento variam de 0 a 100, onde os menores valores indicam melhor percepção do sujeito sobre sua QV e os maiores apontam pior percepção. ${ }^{9,13,23,24}$

\section{Procedimentos}

Os dados foram coletados em quatro etapas: (1) Investigação do banco de dados do Programa Pró-Parkinson, para verificação da elegibilidade dos sujeitos quanto ao interesse em participar da pesquisa, a disponibilidade de estarem duas vezes por semana na pesquisa; (2) Aplicação do PDQ39, em forma de entrevista, para uniformizar a interpretação das perguntas; (3) Intervenção com RVNI; e (4) Reavaliação.

Foram realizadas 20 sessões de RVNI duas vezes por semana, individualmente, com duração entre 30 e 40 minutos cada. Ao final das 20 sessões, os pacientes foram reavaliados com a reaplicação do PDQ-39.

Os equipamentos utilizados na pesquisa durante as intervenções foram: monitor de 40 polegadas LCD, Xbox 360, Kinect, e os jogos Your Shape - Fitness Envolved, Kinect Adventures e Kinect Sports, todos da Microsoft ${ }^{\circledR}$.

As primeiras sessões serviram de treino para que os pacientes aprendessem a acionar o menu do equipamento, escolher os jogos no televisor 
e se posicionar de maneira correta na sala. Ao decorrer das sessões, os jogos eram apresentados.

Os jogos utilizados exigiam dos pacientes mudanças posturais, deslocamento do centro de gravidade corporal, movimentos rápidos e lentos alternados dos MMSS e MMII, além da graduação de força e amplitudes de movimento.

Os equipamentos usados na pesquisa promoveram a interação entre sujeitos e máquina por meio de um sistema de detecção de movimento e representação no vídeo. Um sensor, posicionado junto ao televisor, captou e reproduziu na tela o movimento realizado pelos sujeitos.

$\mathrm{Na}$ sala havia uma pista visual no assoalho identificando a posição correta para que o equipamento escaneasse a imagem dos jogadores e reproduzisse para dentro do ambiente virtual. Assim, o retorno fornecido pela imagem na tela da televisão gerou um reforço positivo, facilitando a formação e o aperfeiçoamento dos exercícios.

Em uma sessão, cada paciente poderia jogar até três diferentes jogos que lhe eram ofertados pelo terapeuta mediante análise da atividade. Foram utilizadas como marcadores de desempenho as pontuações alcançadas e/ou as mudanças de fase a depender da configuração dos jogos.

Análise dos dados

Para análise comparativa dos dados após o término das 20 sessões, foi utilizado o programa estatístico SPSS17. Os testes de normalidade mostraram que os dados não apresentam distribuição normal; desta forma, foi utilizado o teste de Wilcoxon, e considerado um $\mathrm{p}<0,05$.

\section{Aspectos éticos}

Esta pesquisa foi aprovada pelo Comitê de Ética em Pesquisa (CEP) com seres humanos do Centro de Ciências da Saúde da Universidade Federal de Pernambuco (CCS/UFPE), sob protocolo no 00940172000-11. Também foi vinculada à Fundação de Amparo à Ciência e Tecnologia do Estado de Pernambuco (FACEPE) como Iniciação Científica, sob processo n ${ }^{\circ} \mathrm{BIC}$ 0052-4.01/11. Todos os participantes foram esclarecidos quanto aos objetivos da pesquisa e assinaram o Termo de Consentimento Livre e Esclarecido (TCLE).

\section{RESULTADOS}

A amostra foi composta por 14 sujeitos, sendo oito homens e seis mulheres com idades entre 48-84 anos (64tanos). Utilizando a Escala de Estadiamento de HY, pôde-se classificar o grupo da seguinte maneira: estágio I (um paciente); estágio II (cinco pacientes); estágio III (seis pacientes); e estágio IV (dois pacientes).

A tabela 1 mostra as médias dos escores de cada domínio do PDQ-39, assim como do PDQ-39 total, determinadas na avaliação e na reavaliação dos pacientes. Mostra também a diferença absoluta entre esses escores e o valor de $\mathrm{p}$ obtido por meio da análise estatística com o Teste de Wilcoxon, referente à comparação dos escores nos dois momentos. 
Tabela 1. Escores do PDQ-39 antes e após as intervenções. Recife-PE, 2013.

\begin{tabular}{lcccc}
\hline \multirow{2}{*}{ Domínios do PDQ-39 } & \multicolumn{2}{c}{ Escores (média) } & $\begin{array}{c}\text { Diferença absoluta } \\
\text { entre os escores }\end{array}$ & Valor de $\mathrm{p}^{*}$ \\
\cline { 2 - 4 } Mobilidade & Avaliação & Reavaliação & 14 & $0,016^{* *}$ \\
AVD & 43 & 29 & 9 & 0,135 \\
Bem-estar emocional & 37 & 29 & 16 & $0,015^{* *}$ \\
Estigma & 29 & 21 & 16 & $0,017^{* *}$ \\
Suporte social & 6 & 3 & 1 & 0,854 \\
Cognição & 28 & 5 & 12 & $0,038^{* *}$ \\
Comunicação & 14 & 16 & 3 & 0,610 \\
Desconforto corporal & 34 & 11 & 3 & 0,533 \\
\hline PDQ-39 total & 29 & 31 & 10 & $0,009^{* *}$ \\
\hline
\end{tabular}

*Teste de Wilcoxon; **Valores de p estatisticamente significativos.

$\mathrm{Na}$ tabela, verifica-se que os escores das reavaliações foram em média sempre menores do que na avaliação. No entanto, os valores de p mostram que essa diminuição foi significativa $(\mathrm{p}<0,05)$, especificamente para os domínios "mobilidade", "bem-estar emocional", "estigma" e "cognição", ou quando se compara o escore do PDQ-39 como um todo. Já para "AVD”, "suporte social", "comunicação" e "desconforto corporal", não houve diminuição significativa nos escores. Os domínios "bem-estar emocional" e "estigma" obtiveram maiores diferenças absolutas. A "mobilidade" obteve o maior escore na avaliação, entretanto, na reavaliação, este domínio apresenta o segundo maior índice de diferença absoluta. $\mathrm{O}$ "suporte social" quase que permaneceu constante, com diferença de um ponto.

\section{DISCUSSÃO}

A escolha da RV como recurso para intervenção apresenta um crescente uso em diversas áreas, sobretudo na saúde no campo da reabilitação física. ${ }^{15}$ Os atributos da $\mathrm{RV}$ a tornam uma possibilidade para a realização de muitas metas de reabilitação, incluindo o incentivo a aprendizagem para os pacientes, a participação ativa dos mesmos, oferta de ambientes desafiadores, a característica de ser segura, a flexibilidade de individualização e graduação dos protocolos de tratamento, a capacidade para registrar as medidas objetivas de desempenho, além do poder para motivar o paciente a realizar sua capacidade máxima. ${ }^{15}$ 
Os jogos foram escolhidos durante as sessões em conjunto, terapeuta e paciente, considerando o que despertava maior interesse e o que trabalhasse os aspectos que mais incomodassem o paciente. Rand et al. ${ }^{18}$ relatam que a seleção apropriada do material é importante, pois as características do material podem influenciar a maneira como os usuários irão responder ao ambiente virtual.

Os estudos de Silva, Dibai \& Faganello $^{24}$ e Lana et al. ${ }^{22}$ demonstraram que os pacientes com Parkinson apresentavam comprometimento nos domínios "mobilidade" e "AVD" do PDQ-39, cujos escores não diminuíram nos respectivos estudos. No entanto, a presente pesquisa mostrou diminuição dos escores desses domínios, diferindo, assim, do que relatam os autores supracitados.

Vieira et al. ${ }^{15}$ e Silva, Dibai \& Faganello ${ }^{24}$ relatam que esses domínios estão relacionados a atividades de origem motora e que estudos sobre aprendizagem motora em ambiente virtual sugerem que os pacientes com DP apresentam limitações na transferência de aprendizagem do ambiente virtual para o real, acarretando déficits na realização das atividades diárias. Entretanto, Pompeu et al. ${ }^{20}$ e Wang et al., ${ }^{25}$ em seus estudos, relatam que a RV é um meio de intervenção cujas exigências do ambiente virtual estão cada vez mais próximas do real, o que facilitaria a transferência do aprendizado do primeiro ambiente para o segundo.

Wang et al. ${ }^{25}$ discorrem sobre esta capacidade da RV no tocante à velocidade de movimento: pessoas com DP aumentaram a velocidade de movimento em resposta a bolas em movimento rápido no jogo utilizado. Isto sugere que, com uma escolha adequada da velocidade dos alvos, a RV é uma ferramenta promissora para fornecer estímulos visuais em movimento para melhorar a velocidade de movimento, repercutindo na mobilidade e na realização das AVDs. Desta maneira, é importante realizar mais estudos objetivando investigar a relação da realidade virtual e o fazer das atividades no mundo real.

Como relatam alguns autores, a RV caracteriza-se pela rapidez, confiabilidade e capacidade para registrar as medidas objetivas de desempenho, por meio das pontuações. Desta maneira, durante a pesquisa, quando as medidas de desempenho não eram satisfatórias, os pacientes eram estimulados a uma nova tentativa em busca de melhor desempenho. ${ }^{15}$

Estudos relatam que a RV é capaz de melhorar a motivação e, consequentemente, a adesão dos pacientes ao tratamento e o quanto isso reflete sobre o bem-estar dos mesmos, por proporcionar meios de realizarem movimentos corporais desejados e superar os próprios limites na busca por melhor desempenho nos jogos..$^{15,26,27}$ Por ter esse aspecto, a RV pode ter contribuído para a diminuição significativa dos escores no tocante ao domínio "bem-estar emocional".

Os domínios "estigma" e "suporte social" obtiveram diminuição dos escores entre avaliação e reavaliação. No entanto, apenas o primeiro alcançou significância estatística. Para Carod-Artal, Martinez-Martin \& Vargas, ${ }^{28}$ essas dimensões são quase constantes durante a progressão da doença, atribuindo isto à maior divulgação de informações sobre a DP. Todavia, o ambiente em que a pesquisa foi realizada ofertou aos pacientes um espaço de trocas de informações, a fim de estimular a troca de saberes e oportunizar a construção de relações interpessoais.

Os aspectos cognitivos puderam ser trabalhados durante o uso dos jogos, por exemplo, no aprendizado do uso do equipamento, na atenção e concentração exigidas nas partidas, no planejamento e execução dos movimentos a serem realizados, na construção de estratégias para aumentar a pontuação e 
outros, repercutindo, assim, na significância estatística relativa à diminuição do escore desse domínio. Esta perspectiva foi reforçada por um estudo que apresenta a RV como técnica capaz de exercitar as áreas cerebrais referentes a atenção, concentração, percepção visual, orientação espacial, memorização, organização, criatividade, sequência lógica e aprendizagem. ${ }^{29}$

$\mathrm{Na}$ doença de Parkinson, há alterações dos aspectos fonoaudiológicos referentes a mastigação, deglutição, respiração e fonação, que refletem em alterações na qualidade de vida. Os jogos que foram utilizados não exigiam dos pacientes esforços para melhor se comunicarem oralmente, muito menos trabalhar quaisquer aspectos fonoaudiológicos mais específicos, o que pode ter sido a causa do resultado obtido na análise dos escores do domínio "comunicação", que não obteve significância estatística no estudo. ${ }^{30}$

Como no estudo de Carod-Artal, MartinezMartin \& Vargas, ${ }^{28}$ a presente pesquisa também obteve, na análise dos escores, baixo escore total no PDQ-39, o que poderia indicar boa percepção da QV por parte dos pacientes. Considerando a amostra do presente estudo, isto pode ter ocorrido pelo fato de a mesma ter a maioria dos pacientes em estágios menos avançados da doença, conforme a Escala de Hoehn \& Yahr.

Como limitação do estudo destaca-se, em relação ao PDQ-39, a ausência na literatura de um ponto de corte que indique valores mais precisos para representar a boa ou má percepção da QV, o que seria importante para a obtenção de dados mais fidedignos das repercussões dos tratamentos. Talvez a seleção de jogos que exijam a interação dos sujeitos pela comunicação oral pudesse ter modificado o resultado obtido no domínio "comunicação".

Como perspectiva, estudo realizado com um grupo controle, submetido a terapias convencionais, poderá mostrar as diferentes repercussões dos tratamentos por meio dos escores das escalas de avaliação.

Por fim, apesar dos ganhos significativos relatados nesta pesquisa, o estudo apresenta outra limitação - a ausência do follow up para comparar o período de retenção das possíveis melhoras alcançadas pela terapia proposta - não permitindo a análise dos possíveis benefícios da terapia a longo prazo.

\section{CONCLUSÃO}

Neste estudo, o tratamento com realidade virtual não imersiva (RVNI) melhorou a qualidade de vida dos idosos com doença de Parkinson, com ênfase para a percepção de qualidade de vida relacionada ao bem-estar emocional e estigma, seguidos de mobilidade e cognição. Assim, é possível concluir que o enfrentamento da doença de Parkinson deve contemplar, além de seus sinais e sintomas, aspectos relacionados à qualidade de vida, a fim de não subestimar a complexidade do sujeito e a repercussão subjetiva da doença e/ ou tratamento.

O investimento na busca pela evidência dos efeitos do tratamento com RVNI na qualidade de vida na doença de Parkinson precisa continuar, tendo em vista a recente utilização da técnica nessa população. 


\section{REFERÊNCIAS}

1. Camargos ACR, Cópio FCQ, Souza TRR, Goulart F. O impacto da doença de Parkinson na qualidade de vida: uma revisão de literatura. Rev Bras Fisioter 2004;8(3):267-72.

2. Teive HAG. Etiopatogenia da doença de Parkinson. Rev Neurociênc 2005;13(4):201-14.

3. Haase DCBV, Machado DC, Oliveira JGD. Atuação da fisioterapia no paciente com doença de Parkinson. Fisioter Mov 2008;21(1):79-85.

4. Gonçalves GB, Leite MAA, Pereira JS. Influência das distintas modalidades de reabilitação sobre as disfunções motoras decorrentes da Doença de Parkinson. Rev Bras Neurol 2011;47(2):22-30.

5. Almeida MHM, Cruz GA. Intervenções de terapeutas ocupacionais junto a idosos com doença de Parkinson. Rev Ter Ocup 2009;20(1):29-35.

6. Pereira D, Garrett C. Factores de risco da doença de Parkinson: um estudo epidemiológico. Acta Med Port 2010;23(1):15-24.

7. Teive HAG. Neuroproteção: fatos, mitos e quimeras. In: Andrade LAF, Barbosa ER, Cardoso F, Teive HAG. Doença de Parkinson: estratégias atuais de tratamento. 2. ed. São Paulo: Segmento Farma; 2006. p. 17-35.

8. Almeida MHM, Castiglioni MC. Recursos tecnológicos: estratégia de promoção do autocuidado, atividades e participação para pessoas com doença de Parkinson. Rev Ter Ocup 2007;18(3):152-7.

9. Marinus J, Ramaker C, Van Hilten JJ, Stiggelbout AM. Health related quality of life in Parkinson's disease: a systematic review of disease specific instruments. J Neurol Neurosurg Psychiatry 2002;72(2):241-8.

10. Seidl EMF, Zannon CMLC. Qualidade de vida e saúde: aspectos concei $\neg$ tuais e metodológicos. Cad Saúde Pública 2004;20(2):580-8.

11. Goulart F, Pereira LX. Uso de escalas para avaliação da doença de Parkinson em fisioterapia. Fisioter Pesqui 2005;2(1):49-56.

12. Scattolin FAA. Qualidade de vida: a evolução do conceito e os instrumentos de medida. Rev Fac Ciênc Méd Sorocaba 2006;8(4):1-5.
13. Navarro-Peternella FM, Marcon SS. Qualidade de vida de indivíduos com Parkinson e sua relação com tempo de evolução e gravidade da doença. Rev Latinoam Enferm 2012;20(2):1-8.

14. Findley LJ. The economic impact of Parkinson's disease. Parkinsonism Relat Disord 2007;13 Suppl:8-12.

15. Vieira GP, Araújo DFGH, Leite MAA, Orsini M, Correa CL. Realidade virtual na reabilitação física de pacientes com Doença de Parkinson. J Hum Growth Dev 2014;24(1):31-41.

16. Adamovich SV, Fluet GG, Tunik E, Merians AS. Sensorimotor training in virtual reality: a review. NeuroRehabilitation 2009;25(1):29-44.

17. Kirner C, Siscoutto RA. Fundamentos de realidade virtual e aumentada. In: Siscoutto RA, Costa R. Realidade virtual e aumentada: uma abordagem tecnológica. Porto Alegre: Sociedade Brasileira de Computação; 2008. p. 1-20.

18. Rand D, Kizony R, Feintuch U, Katz N, Josman N, Rizzo AS, et al. Comparison of two VR platforms for rehabilitation: video capture versus HMD. Presence 2005;14(2):147-60.

19. Tori R, Kirner C. Fundamentos da realidade virtual. In: Tori R, Kirner C, Siscoutto R, editores. Fundamentos e tecnologia de realidade virtual e aumentada. Porto Alegre: Sociedade Brasileira de Computação; 2006. p. 2-23.

20. Pompeu JE, Mendes FAS, Silva KG, Lobo AM, Oliveira TP, Zomignani AP, et al. Effect of Nintendo Wii ${ }^{{ }^{T M}}$-based motor and cognitive training on activities of daily living in patients with Parkinson's disease: a randomised clinical trial. Physiotherapy 2012;98(3):196-204.

21. Pedral C,Bastos PA. Terapia ocupacional: metodologia e prática. 2. ed. Rio de Janeiro: Rubio; 2013.

22. Lana RC, Álvares LMRS, Nasciutti-Prudente C, Goulart FRP, Teixeira-Salmela LF, Cardoso FE. Percepção da qualidade de vida de indivíduos com doença de Parkinson através do PDQ-39. Rev Bras Fisioter 2007;11(5):397-402. 
23. Souza RG, Borges V, Silva SMCA, Ferraz HB. Quality of life scale in parkinson's disease PDQ-39: (Brazilian Portuguese version) to assess patients with and without levodopa motor fluctuation. Arq Neuropsiquiatr 2007;65(3b):787-91.

24. Silva JAMG, Dibai AV Filho, Faganello FR. Mensuração da qualidade de vida de indivíduos com a doença de Parkinson por meio do questionário PDQ39. Fisioter Mov 2011;24(1):141-6.

25. Wang CY, Hwang WJ, Fang JJ, Sheu CF, Leong IF, Ma HI. Comparison of virtual reality vesus Physical reality on movement characteristics of persons with Parkinson's disease: effects of moving targets. Arch Phys Med Rehabil 2011;92(8):1238-45.

26. Mendes FA, Pimpeu JE, Lobo AM, Silva KG, Oliveira TP, Zomignani AP, et al. Motor learning, retention and transfer after virtual-reality-based training in Parkinson's disease-effect of motor and cognitive demands of games: a longitudinal, controlled clinical study. Physiotherapy 2012;98(3):217-23.
27. Dias RS, Sampaio ILA, Taddeo LS. Fisioterapia X WII: a introdução do lúdico no processo de reabilitação de pacientes em tratamento fisioterápico. In: Sociedade Brasileira de computação. $8^{\circ}$ Brazilian Symposium on Games and Digital Entertainment; 08-10 Out 2009; Rio de Janeiro: SBgaes; 2009.

28. Carod-Artal FJ, Martinez-Martin P, Vargas AP. Independent validation of SCOPA-Psychosocial and metric properties of the PDQ-39 brasilian version. Mov Disord 2007;22(1):91-8.

29. Jerônimo RA, Lima SMPF. Tecnologias computacionais e ambientes virtuais no processo terapêutico de reabilitação. Mundo Saúde 2006;30(1):96-106.

30. Schilling GR, Hilbig A, Cardoso MC. Aspectos fonoaudiológicos e qualidade de vida na Doença de Parkinson: estudos de Casos. Rev Geriatr Gerontol 2014;9(2):124-31. 